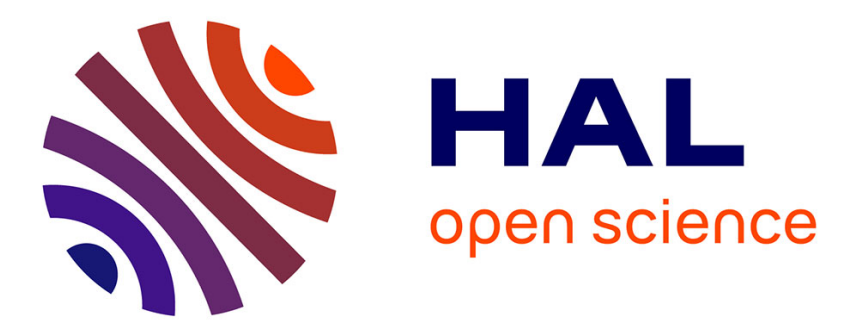

\title{
Characterization of Progressive Fatigue Damage in Solid Plates by Laser Ultrasonic Monitoring of Zero-Group-Velocity Lamb Modes
}

Guqi Yan, Samuel Raetz, Nikolay Chigarev, Vitali Goussev, Vincent Tournat

\section{- To cite this version:}

Guqi Yan, Samuel Raetz, Nikolay Chigarev, Vitali Goussev, Vincent Tournat. Characterization of Progressive Fatigue Damage in Solid Plates by Laser Ultrasonic Monitoring of Zero-Group-Velocity Lamb Modes. Physical Review Applied, 2018, 9 (6), 10.1103/PhysRevApplied.9.061001 . hal-01889443

HAL Id: hal-01889443

https://hal-univ-lemans.archives-ouvertes.fr/hal-01889443

Submitted on 9 Oct 2018

HAL is a multi-disciplinary open access archive for the deposit and dissemination of scientific research documents, whether they are published or not. The documents may come from teaching and research institutions in France or abroad, or from public or private research centers.
L'archive ouverte pluridisciplinaire $\mathbf{H A L}$, est destinée au dépôt et à la diffusion de documents scientifiques de niveau recherche, publiés ou non, émanant des établissements d'enseignement et de recherche français ou étrangers, des laboratoires publics ou privés. 


\title{
Characterization of Progressive Fatigue Damage in Solid Plates by Laser Ultrasonic Monitoring of Zero-Group-Velocity Lamb Modes
}

\author{
Guqi Yan, Samuel Raetz, Nikolay Chigarev, Vitalyi E. Gusev, and Vincent Tournat ${ }^{\dagger}$ \\ LAUM, Le Mans Université, UMR CNRS 6613, Avenue Olivier Messiaen, 72085 Le Mans Cedex 9, France
}

(Received 15 December 2017; revised manuscript received 27 April 2018; published 19 June 2018)

\begin{abstract}
We propose a method for nondestructive characterization of progressive fatigue damage in solid plates, using a zero-group-velocity (ZGV) Lamb mode generated and detected by lasers. Our experimental results depict a nonmonotonous change in the ZGV mode frequency with an increasing number of loading cycles, and more importantly its drastic decrease prior to the specimen failure. We report three experiments on three specimens made of aluminum, which fail after different numbers of loading cycles. Despite this difference, the three nonmonotonous variations of the ZGV mode frequency with an increasing number of loading cycles are superimposed when plotted as a function of the normalized fatigue lifetime. This feature stresses out the potential of the technique to locate fatigue damage, to predict the fatigue lifetime, and to qualitatively, and even quantitatively, assess the different stages of fatigue damage in micrometer- to potentially centimeters-thick solid plates.
\end{abstract}

DOI: 10.1103/PhysRevApplied.9.061001

\section{INTRODUCTION}

Solid structures, such as metallic sheets, are generally subjected during their lifetime to numerous stresses (mechanical, thermal, chemical, neutron-ion irradiation), causing fatigue and leading inexorably to damage or even failure of the specimen. It is therefore of utmost importance, in many industrial contexts, to nondestructively assess the cumulative fatigue damage in solid structures used in manufactured products, for their reliability, quantification of health, and certification of security. This challenge has been continuously recalled to be of scientific importance over the last decade [1-5].

Ultrasonic methods were often used for fatigue assessments by detecting acoustic nonlinearity, crack length, elasticity, and the accumulation of damage [6-12]. Laser ultrasonic (LU) techniques have demonstrated increasingly high capabilities in the nondestructive evaluation (NDE) of the mechanical properties of materials [13-21]. Their specific advantages, such as high spatial resolution, large bandwidth, and noncontact character, when compared to conventional ultrasonic methods based on piezoelectric transducers, are particularly appealing. In parallel, the recently implemented laser-based zero-group-velocity (ZGV) Lamb modes have proven to be an efficient tool to probe locally and accurately the thickness or the mechanical properties of plates, as well as to detect defects [22-29]. Yet, the use of LU monitoring of ZGV Lamb modes in solid plates, in order to evaluate or image the sample's damage, especially the fatigue-induced

\footnotetext{
*samuel.raetz@univ-lemans.fr

†vincent.tournat@univ-lemans.fr
}

damage, has not been reported, while it could be useful for both modeling developments and practical applications.

Over the last three decades, much research has been dedicated to proposing measurable physical parameters informative of the fatigue stage of a material, subjected to fatigue test. In this frame, parameters such as damage index, Young's modulus, density, and cross-sectional area, have demonstrated changes with respect to the fatigue lifetime [30-37]. However, the variation of these parameters were all reported to be monotonous (either decreasing or increasing) with the fatigue of the material, which necessitates somehow a definition (often rather arbitrary) of a quantitative threshold denoting a given fatigue stage. Therefore, an index which shows a robust nonmonotonous trend as a function of the amount of loading cycles or fatigue lifetime, would have the important advantage of defining by itself a threshold in the fatigue process, easily detected and surely related to the onset or domination of specific physical processes.

We report here on the monitoring of ZGV Lamb mode frequency along fatigue damage progression in a solid plate made of aluminum. Specifically, the change in the frequency of the first symmetrical ZGV Lamb mode is analyzed as a function of both the fatigue lifetime and the probing location on the sample. In addition to a spatial image of the damage, we observe a reproducible nonmonotonous trend in the evolution of the $\mathrm{ZGV}$ mode frequency, with a single extremum occurring consistently for different tests at about $80 \%$ of the fatigue lifetime. The thickness of the here-tested plates is $75 \mu \mathrm{m}$. Yet, the technique is sound for micrometerthick-to-centimeters-thick plates, depending on the pulsed generation laser characteristics (pulsed duration, energy). 


\section{ZERO-GROUP-VELOCITY LAMB MODES}

Lamb waves are the acoustic eigenmodes guided in a freestanding plate [38-40]. A ZGV Lamb mode results from the interference of two Lamb waves coexisting for a unique couple of frequency and wave number but spatially counterpropagating. This specific type of Lamb mode exhibits an anomalous behavior since the group velocity $V_{g}=d \omega / d k$ vanishes, while the phase velocity remains finite. The mechanical energy associated with a ZGV mode is, therefore, locally trapped at the excitation area, which leads to a sharp resonance of the plate. Because of the local feature of this resonance, its frequency value is informative of the local mechanical properties or thickness. For a single homogeneous plate, the Rayleigh-Lamb equations can be written as a function of the dimensionless frequency $\Omega=2 h \omega /$ $\left(\pi c_{T}\right)$ and the dimensionless wave number $\xi=2 \mathrm{kh} / \pi$, where $2 h$ stands for the plate thickness, $\omega$ for the pulsation, $c_{T}$ for the shear wave velocity, and $k$ for the wave number [38-40]. Therefore, if the elastic parameters of the plate remain unchanged, the frequency shift $\Delta f(\alpha \Delta \omega)$ of the $\mathrm{ZGV}$ mode is associated with the change of the plate thickness $\Delta h$ :

$$
\frac{\Delta f}{f}=-\frac{\Delta h}{h}
$$

The relation (1) is useful for NDE purposes [29,41-43] and will be used in the following to explain the experimentally revealed phenomena. It is also important to note that the spatial resolution of our method is scalable with the plate thickness since the $\mathrm{ZGV}$ mode wavelength is of the order of twice the plate thickness. This feature, therefore, probably limits the potential of our technique to detect small fatigue zones in a centimeters-thick plate.

\section{FATIGUE SETUP AND LASER ULTRASONIC EXPERIMENTAL SETUP}

In this Letter, a homemade fatigue machine is used for cyclic loading of a $75-\mu \mathrm{m}$-thick aluminium plate in a twosides-clamped compression configuration inducing buckling [see Fig. 1(c)]. The buckling is achieved by imposing one of the clamped end of the plate of length $2 L$ to move by $2 \Delta L$ along the $x$ axis [see Fig. 1(c)]. The analytical expression of the normal deflection $w(x)$ of the plate neutral line in this buckling configuration is [44]

$$
w(x)=\frac{\delta}{2}\left[\cos \left(\frac{\pi(x-L+\Delta L)}{L-\Delta L}\right)-1\right],
$$

where $\delta$ is the buckling deflection at the center of the specimen $(x=0)$. From Eq. (2), the stress $\sigma_{x x}(x, z)$ is then readily deduced [45]:

$$
\sigma_{x x}(x, z)=A z \cos \left(\frac{\pi(x-L+\Delta L)}{L-\Delta L}\right),
$$
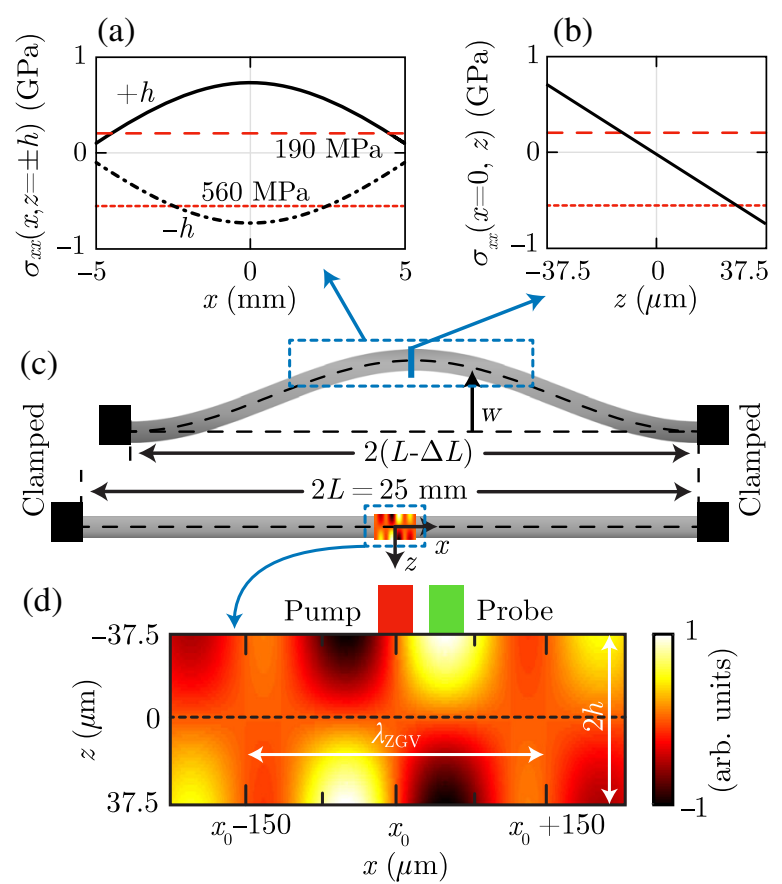

FIG. 1. (a) Surface $(z= \pm h)$ stress profiles of the buckled solid plate and (b) section (at $x=0 \mathrm{~mm}$ ) stress distribution with $\Delta L=2 \mathrm{~mm}$. (c) Schematic presentation of the loaded and unloaded plate. (d) $x$ component of the normal displacement gradient $\left(\partial u_{z} / \partial x\right)$ associated with the first symmetrical ZGV Lamb mode $(2 h=75 \mu \mathrm{m})$.

where $A=-\delta \pi^{2} E /\left[2(L-\Delta L)^{2}\left(1-\nu^{2}\right)\right], E$ and $\nu$ standing for the Young's modulus and the Poisson's ratio, respectively. Here, the zero of the local $z=z(x)$ axis, normal to the plate surfaces, is chosen in the middle plane of the plate. In our experiment, the length of the plate is $2 L=$ $25 \mathrm{~mm}$ and the imposed $x$ displacement is $2 \Delta L=4 \mathrm{~mm}$, leading to a buckling deflection of $\delta=5.83 \mathrm{~mm}$. In that case, and using $E=70 \mathrm{GPa}$ and $\nu=0.35$ (corresponding to aluminum), the stress distribution at $z= \pm h$ is plotted in Fig. 1(a) as a function of $x$. The red dashed line and dotted line represent the tensile (approximately $190 \mathrm{MPa}$ ) and compressive (approximately $560 \mathrm{MPa}$ ) yield strengths of the tested aluminum specimen, respectively. Please note that the compressive yield strength has been taken from p. 152 of Ref. [46]. From Fig. 1(a), it can be stated that the most fatigued part of the plate is expected to be located at $x=0$, since the maximum difference between the applied stress and the tensile-compressive yield stress occurs at this location [see Figs. 1(a) and 1(b)]. Note that the fatigue could be expected for all points from $x=0$ to $x \approx \pm 5 \mathrm{~mm}$ with a continuous decrease in magnitude since the applied stresses approach the yield stresses as the distance from $x=0 \mathrm{~mm}$ increases. This low-cycle fatigue is in our case most probably related to the propagation of surface flaws than to dislocation mechanisms. In order to perform the measurements of the variation of the ZGV mode frequency along the fatigued plate, the loading is stopped at regular intervals of cycles. The 
scan of the unloaded plate in front of the laser ultrasonic setup is performed over the interval $x \in[-5 ; 5] \mathrm{mm}$, with a scan step of $100 \mu \mathrm{m}$, using a motorized actuator with a minimum incremental motion of $0.1 \mu \mathrm{m}$.

The laser ultrasonic experimental setup used to monitor the ZGV mode frequency is composed of (i) a pulsed $\mathrm{Nd}$ : YAG laser $(1064 \mathrm{~nm}$, pulse duration close to $0.75 \mathrm{~ns}$, repetition rate of $1 \mathrm{kHz}$ ) focused to a stretched ellipse (small diameter approximately $20 \mu \mathrm{m}$ and large diameter approximately $500 \mu \mathrm{m}$ ) on the sample surface to generate the first symmetrical ZGV Lamb mode among others, and (ii) a $\mathrm{CW}$ laser $(532 \mathrm{~nm})$ focused to a circular spot (diameter approximately $20 \mu \mathrm{m}$ ) in the vicinity of the line source to detect the local resonance thanks to the beamdeflection technique [20,47]. Since the beam-deflection technique is sensitive to the radial gradient of the normal displacement $\left(u_{z}\right)$, a small shift of about $40 \mu \mathrm{m}$ has been used between generation and detection spots, for the detection of the ZGV mode. This is illustrated in Fig. 1(d), also showing the distribution of the $x$ component of the normal displacement gradient $\left(\partial u_{z} / \partial x\right)$ associated with the first symmetrical ZGV Lamb mode. Note that the specimen is unloaded at regular intervals of 500 cycles in the conducted experiments and that a reference scan has been achieved on the intact specimen and saved for comparison with results at different fatigue stages.

\section{RESULTS AND DISCUSSION}

An example of a measured temporal photoacoustic signal is displayed in Fig. 2(a). The slowly oscillating background corresponds to the first antisymmetric mode $A_{0}$ which is preponderant. By enlarging the time signal [inset in Fig. 2(a)], a higher frequency oscillation corresponding to the ZGV resonance is visible. The Fourier transform (FT) of the signal in Fig. 2(a) is calculated and its normalized magnitude with respect to the $\mathrm{ZGV}$ peak value is plotted in the frequency range of $20-100 \mathrm{MHz}$ in Fig. 2(b). A resonance is visible at $38.78 \mathrm{MHz}$, having a measured quality factor of 149. In Fig. 2(c), the red solid line exhibits the spatial distribution of the $\mathrm{ZGV}$ resonance frequency after 9500 fatigue loading cycles, which is extracted from the normalized FT magnitude. A very clear frequency increase is observed in the center of the sample (maximum increase at position $x=0.1 \mathrm{~mm}$ ), primarily due to the local decrease of the thickness in this fatigued region.

The relative variation $\Delta f / f$ of the ZGV frequency measured after $N$ cycles compared to the reference ZGV frequency (measured on the intact specimen) is calculated as a function of the sample position and of the number of loading cycles and displayed in Fig. 3(a). Three main results emerge from this plot: (i) the obvious distinction on ZGV frequency between damaged and undamaged regions; (ii) the monotonic increase of $\mathrm{ZGV}$ frequency in the fatigue region up to 10000 cycles, mainly caused by thickness changes, as expected from Eq. (1); (iii) the abrupt decrease of the ZGV
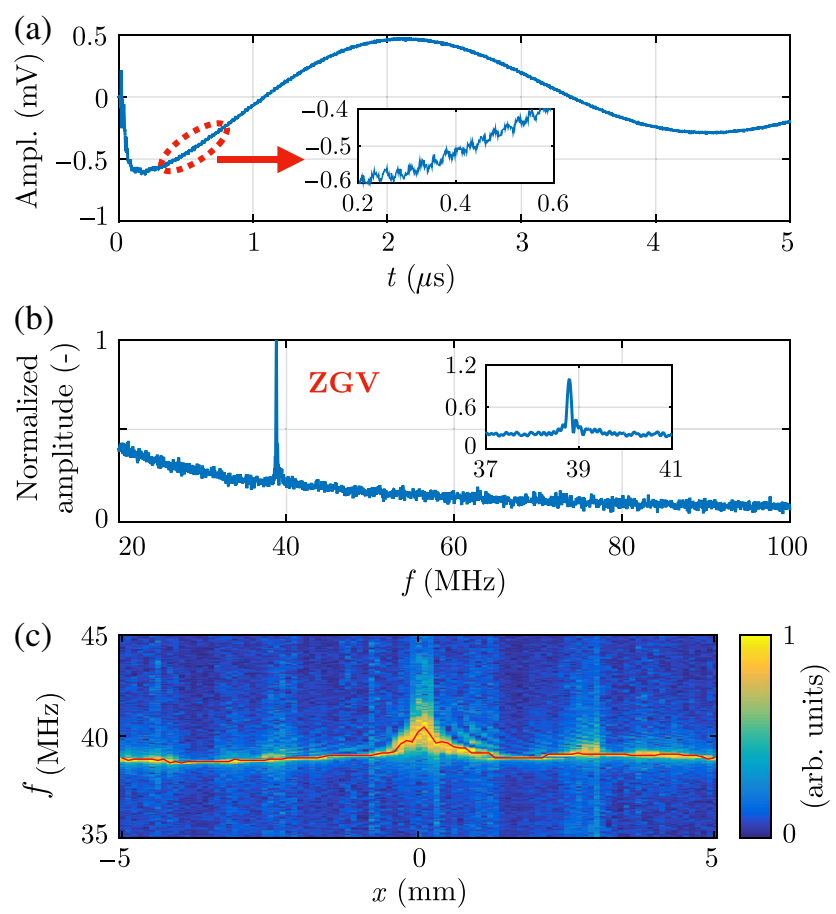

FIG. 2. (a) Example of a received temporal photoacoustic signal measurement and (b) its spectrum with a sharp peak corresponding to the ZGV resonance frequency. (c) Spectrum magnitude normalized by the $\mathrm{ZGV}$ peak value as a function of frequency and sample position after 9500 fatigue loading cycles, the red solid line represents the spatial distribution of the estimated $\mathrm{ZGV}$ resonance frequency.

frequency before specimen failure. For a better appreciation of this last point, the variation of the ZGV frequency at $x=0.1 \mathrm{~mm}$ is extracted from the image and is plotted in Fig. 3(b); error bars stand for $f_{n} /\left(f_{0} Q_{n}\right)$, with $f_{n}$ the ZGV frequency after $n$ cycles, $Q_{n}$ the $\mathrm{Q}$ factor of the corresponding resonance. The maximum value at 10000 loading cycles divides the curve into two different parts: (i) the monotonic increase where the thickness change is mainly at play and (ii) the abrupt decrease before specimen failure that could be explained by the drop in elastic stiffness $[30,48]$ and by the interaction between the ZGV mode and the dislocations and cracks present in the specimen [12,49-51]. Note that fatigue softening of materials can be found not only in metals [37,52], but also in other types of materials, such as polymer [6], composite materials [53], and so on.

Following these experimental results, it can be stated that the proposed method is sensitive to cumulative fatigue damage [see Figs. 2(c) and 3(a)]. Moreover, even without knowing the exact position of the damaged zone before observation, which could be a limitation of some other methods [35,54], it can be located thanks to the frequency image as depicted in Fig. 2(c). Last but not least, unlike other damage indexes presented in the literature [6,9,30-37,48], the nonmonotonic evolution of the variation $\Delta f / f$ of the ZGV resonance frequency shows the potential for quantitative assessment of the fatigue lifetime [see Fig. 3(b)]. Even 


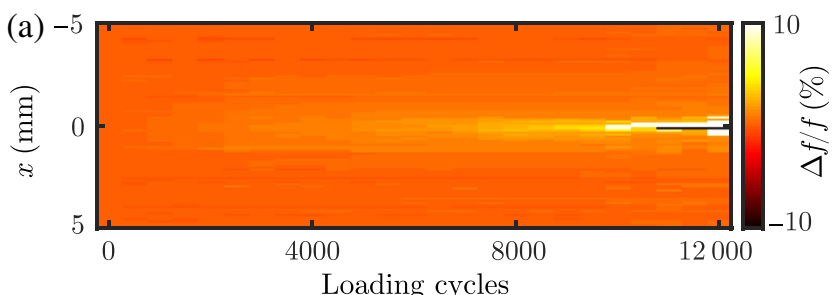

Loading cycles
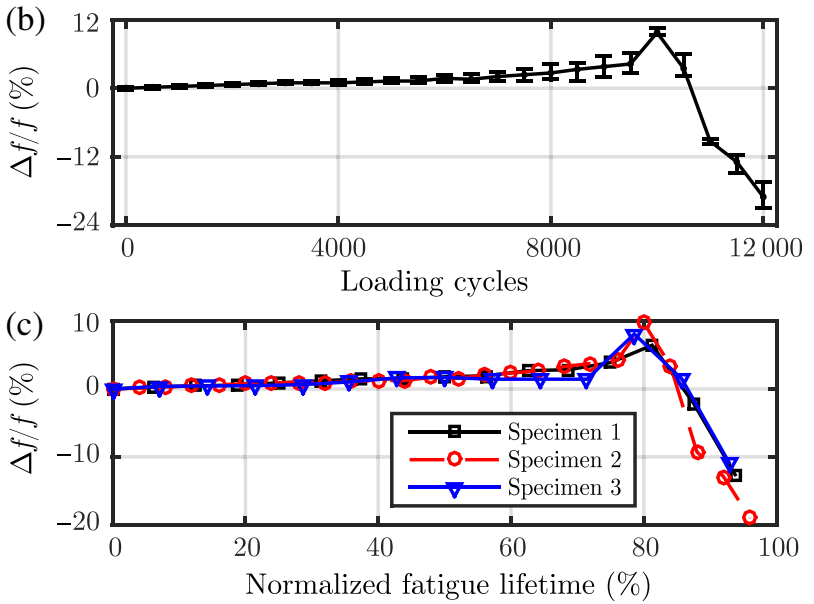

FIG. 3. (a) Spatial distribution of the relative variation of the ZGV mode frequency vs fatigue cycles. (b) Experimental evolution of $\Delta f / f$ (with error bars) as a function of fatigue duration at the specimen center $(x=0.1 \mathrm{~mm})$. (c) Comparison between $\Delta f / f$ evolutions measured in specimens 1,2 , and 3 (failure at approximately 16000,12500 , and 14000 cycles) as a function of normalized fatigue lifetime.

if ascertaining which side of the peak corresponds to the correct measurement of the fatigue state might be seen as a limitation of our technique when the material is not continuously monitored, two consecutive measurements of this frequency at a small fatigue cycle interval would suffice to ascertain to which side of the peak belongs the current state. Overcoming this limitation is currently under development and should be addressed in follow-up papers. Three fatigue monitoring tests have been carried out on three samples and the corresponding $\Delta f / f$ are plotted in Fig. 3(c), showing identical variations in normalized fatigue lifetime scale, although failure occurs at different amounts of loading cycles. This reproducible, nonmonotonous parameter variation [see Fig. 3(c)], thanks to the existence of an extremum just prior to the fracture of the specimen (approximately 80\% of the fatigue lifetime), might establish a quantitative empirical law characterizing a sample's fatigue stage and could be an effective tool for predicting fatigue life.

\section{CONCLUSION}

In conclusion, this Letter presents a noncontact laser ultrasonics evaluation of cumulative fatigue damage in thin solid plates by monitoring a ZGV Lamb mode frequency. The experimental results demonstrate the ability of this method for (i) locating damage, (ii) predicting fatigue lifetime, and (iii) assessing qualitatively and potentially quantitatively, the cumulative fatigue damage levels during fatigue process. For better understanding of elastic property changes and of thickness distribution in the specimen, numerical simulations (based on the finite element method, for example) are necessary and are under development. One of the prospects of this work is to formally identify the material mechanisms leading to this extremum in $\mathrm{ZGV}$ Lamb mode frequency using, e.g., electron microscope imaging, in order to reach a fully quantitative (local) damage evaluation of a sample [55,56].

[1] P. J. Withers, Residual stress and its role in failure, Rep. Prog. Phys. 70, 2211 (2007).

[2] J. J. Kruzic, Predicting fatigue failures, Science 325, 156 (2009).

[3] P. Li, S. X. Li, Z. G. Wang, and Z. F. Zhang, Fundamental factors on formation mechanism of dislocation arrangements in cyclically deformed fcc single crystals, Prog. Mater. Sci. 56, 328 (2011).

[4] A. Pineau, D. L. McDowell, E. P. Busso, and S. D. Antolovich, Failure of metals II: Fatigue, Acta Mater. 107, 484 (2016).

[5] M. Kamal and M. M. Rahman, Advances in fatigue life modeling: A review, Renew. Sustain. Energ. Rev. 82, 940 (2018).

[6] P. B. Nagy, Fatigue damage assessment by nonlinear ultrasonic materials characterization, Ultrasonics 36, 375 (1998).

[7] C. Bermes, J. Kim, J. Qu, and L. J. Jacobs, Experimental characterization of material nonlinearity using Lamb waves, Appl. Phys. Lett. 90, 021901 (2007).

[8] J. H. Cantrell and W. T. Yost, Nonlinear ultrasonic characterization of fatigue microstructures, Int. J. Fatigue 23, 487 (2001).

[9] M. Deng and J. Pei, Assessment of accumulated fatigue damage in solid plates using nonlinear Lamb wave approach, Appl. Phys. Lett. 90, 121902 (2007).

[10] V. V. S. Jaya Rao, E. Kannan, R. V. Prakash, and K. Balasubramaniam, Fatigue damage characterization using surface acoustic wave nonlinearity in aluminum alloy AA7175-T7351, J. Appl. Phys. 104, 123508 (2008).

[11] C. Pruell, J. Kim, J. Qu, and L. J. Jacobs, Evaluation of plasticity driven material damage using Lamb waves, Appl. Phys. Lett. 91, 231911 (2007).

[12] L. J. Pyrak-Nolte, J. Xu, and G. M. Haley, Elastic Interface Waves Propagating in a Fracture, Phys. Rev. Lett. 68, 3650 (1992).

[13] C. B. Scruby and L. E. Drain, Laser Ultrasonics Techniques and Applications (Taylor \& Francis, London, 1990).

[14] D. Royer and E. Dieulesaint, Elastic Waves in Solids II: Generation, Acousto-optic Interaction, Applications, Advanced Texts in Physics Vol. II (Springer, Berlin, Heidelberg, 2000).

[15] P. Hess, Surface acoustic waves in materials science, Phys. Today 55, No. 3, 42 (2002).

[16] A. K. Kromine, P. A. Fomitchov, S. Krishnaswamy, and J.D. Achenbach, Laser ultrasonic detection of surface breaking discontinuities: Scanning laser source technique, 
Mater. Eval. 58, 173 (2000), https://www.scholars .northwestern.edu/en/publications/laser-ultrasonic-detectionof-surface-breaking-discontinuities-sc.

[17] S. Dixon, B. Cann, D. L. Carroll, Y. Fan, and R. S. Edwards, Non-linear enhancement of laser generated ultrasonic Rayleigh waves by cracks, Nondestr. Test. Eval. 23, 25 (2008).

[18] C. Thomsen, J. Strait, Z. Vardeny, H. J. Maris, J. Tauc, and J. J. Hauser, Coherent Phonon Generation and Detection by Picosecond Light Pulses, Phys. Rev. Lett. 53, 989 (1984).

[19] Al. A. Kolomenskii, A. M. Lomonosov, R. Kuschnereit, P. Hess, and V. E. Gusev, Laser Generation and Detection of Strongly Nonlinear Elastic Surface Pulses, Phys. Rev. Lett. 79, 1325 (1997).

[20] S. Mezil, N. Chigarev, V. Tournat, and V. Gusev, All-optical probing of the nonlinear acoustics of a crack, Opt. Lett. 36, 3449 (2011).

[21] C. Ni, N. Chigarev, V. Tournat, N. Delorme, Z. Shen, and V.E. Gusev, Probing of laser-induced crack closure by pulsed laser-generated acoustic waves, J. Appl. Phys. 113, 014906 (2013).

[22] C. Prada, O. Balogun, and T. W. Murray, Laser-based ultrasonic generation and detection of zero-group velocity Lamb waves in thin plates, Appl. Phys. Lett. 87, 194109 (2005).

[23] D. Clorennec, C. Prada, D. Royer, and T. W. Murray, Laser impulse generation and interferometer detection of zero group velocity Lamb mode resonance, Appl. Phys. Lett. 89, 024101 (2006).

[24] D. Clorennec, C. Prada, and D. Royer, Local and noncontact measurements of bulk acoustic wave velocities in thin isotropic plates and shells using zero group velocity Lamb modes, J. Appl. Phys. 101, 034908 (2007).

[25] S. Mezil, F. Bruno, S. Raetz, J. Laurent, D. Royer, and C. Prada, Investigation of interfacial stiffnesses of a tri-layer using zero-group velocity Lamb modes, J. Acoust. Soc. Am. 138, 3202 (2015).

[26] C. Prada, D. Clorennec, and D. Royer, Local vibration of an elastic plate and zero-group velocity Lamb modes, J. Acoust. Soc. Am. 124, 203 (2008).

[27] C. Prada, D. Clorennec, T. W. Murray, and D. Royer, Influence of the anisotropy on zero-group velocity Lamb modes, J. Acoust. Soc. Am. 126, 620 (2009).

[28] S. Raetz, J. Laurent, T. Dehoux, D. Royer, B. Audoin, and C. Prada, Effect of refracted light distribution on the photoelastic generation of zero-group velocity lamb modes in optically low-absorbing plates, J. Acoust. Soc. Am. 138, 3522 (2015).

[29] F. Faëse, S. Raetz, N. Chigarev, C. Mechri, J. Blondeau, B. Campagne, V. E. Gusev, and V. Tournat, Beam shaping to enhance zero group velocity Lamb mode generation in a composite plate and nondestructive testing application, NDT\&E Int. 85, 13 (2017).

[30] A. Plumtree and B. P. D. O'Connor, Damage accumulation and fatigue crack propagation in a squeeze-formed aluminium alloy, Int. J. Fatigue 11, 249 (1989).

[31] A. Fatemi and L. Yang, Cumulative fatigue damage and life prediction theories: A survey of the state of the art for homogeneous materials, Int. J. Fatigue 20, 9 (1998).

[32] B. Sun, L. Yang, and Y. Guo, A high-cycle fatigue accumulation model based on electrical resistance for structural steels, Fatigue Fract. Eng. Mater. Struct. 30, 1052 (2007).

[33] X. J. Sun, C. C. Wang, J. Zhang, G. Liu, G. J. Zhang, X. D. Ding, G. P. Zhang, and J. Sun, Thickness dependent fatigue life at microcrack nucleation for metal thin films on flexible substrates, J. Phys. D 41, 195404 (2008).

[34] J. H. Cantrell, Ultrasonic harmonic generation from fatigueinduced dislocation substructures in planar slip metals and assessment of remaining fatigue life, J. Appl. Phys. 106, 093516 (2009).

[35] M. Amura, M. Meo, and F. Amerini, Baseline-free estimation of residual fatigue life using a third order acoustic nonlinear parameter, J. Acoust. Soc. Am. 130, 1829 (2011).

[36] A. Aid, A. Amrouche, B. Bachir Bouiadjra, M. Benguediab, and G. Mesmacque, Fatigue life prediction under variable loading based on a new damage model, Mater. Des. 32, 183 (2011).

[37] S. J. Li, L. E. Murr, X. Y. Cheng, Z. B. Zhang, Y. L. Hao, R. Yang, F. Medina, and R. B. Wicker, Compression fatigue behavior of Ti-6Al-4V mesh arrays fabricated by electron beam melting, Acta Mater. 60, 793 (2012).

[38] J. D. Achenbach, Wave Propagation in Elastic Solids (North-Holland Publishing Company, Amsterdam, 1973).

[39] B. A. Auld, Acoustic Fields and Waves in Solids (R. E. Krierger Publishing Company, Malabar, FL, 1990), Vol. 2.

[40] D. Royer and E. Dieulesaint, Elastic Waves in Solids (Masson, Paris, 1999), Vol. 1.

[41] D. Clorennec, C. Prada, and D. Royer, Laser ultrasonic inspection of plates using zero-group velocity Lamb modes, IEEE Trans. Ultrason. Ferroelectr. Freq. Control 57, 1125 (2010).

[42] M. Cès, D. Clorennec, D. Royer, and C. Prada, Thin layer thickness measurements by zero group velocity Lamb mode resonances, Rev. Sci. Instrum. 82, 114902 (2011).

[43] M. Cès, D. Royer, and C. Prada, Characterization of mechanical properties of a hollow cylinder with zero group velocity Lamb modes, J. Acoust. Soc. Am. 132, 180 (2012).

[44] J. W. Hutchinson, Stresses and Failure Modes in Thin Films and Multilayers (Technical University of Denmark, Denmark, 1996).

[45] H. Chai and C. D. Babcock, Two-dimensional modelling of compressive failure in delaminated laminates, J. Compos. Mater. 19, 67 (1985).

[46] N. E. Dowling, K. S. Prasad, and R. Narayanasamy, Mechanical Behavior of Materials: Engineering Methods for Deformation, Fracture, and Fatigue (Pearson, Essex, England, 2012).

[47] J. Higuet, T. Valier-Brasier, T. Dehoux, and B. Audoin, Beam distortion detection and deflectometry measurements of gigahertz surface acoustic waves, Rev. Sci. Instrum. 82, 114905 (2011).

[48] J. Lemaitre and J. Dufailly, Damage measurements, Eng. Fract. Mech. 28, 643 (1987).

[49] A. Maurel, V. Pagneux, F. Barra, and F. Lund, Wave propagation through a random array of pinned dislocations: Velocity change and attenuation in a generalized Granato and Lücke theory, Phys. Rev. B 72, 174111 (2005).

[50] N. Mujica, M. T. Cerda, R. Espinoza, J. Lisoni, and F. Lund, Ultrasound as a probe of dislocation density in aluminum, Acta Mater. 60, 5828 (2012). 
[51] F. Barra, R. Espinoza-González, H. Fernández, F. Lund, A. Maurel, and V. Pagneux, The use of ultrasound to measure dislocation density, JOM 67, 1856 (2015).

[52] M. W. Barsoum, M. Radovic, T. Zhen, P. Finkel, and S. R. Kalidindi, Dynamic Elastic Hysteretic Solids and Dislocations, Phys. Rev. Lett. 94, 085501 (2005).

[53] N. Rauter, R. Lammering, and T. Kühnrich, On the detection of fatigue damage in composites by use of second harmonic guided waves, Compos. Struct. 152, 247 (2016).
[54] M. Amura and M. Meo, Prediction of residual fatigue life using nonlinear ultrasound, Smart Mater. Struct. 21, 045001 (2012).

[55] D. Farkas, M. Willemann, and B. Hyde, Atomistic Mechanisms of Fatigue in Nanocrystalline Metals, Phys. Rev. Lett. 94, 165502 (2005).

[56] M. A. Meyers, A. Mishra, and D. J. Benson, Mechanical properties of nanocrystalline materials, Prog. Mater. Sci. 51, 427 (2006). 